\title{
Skirt Corrections in the Variable Pressure SEM Related to Rutherford Cross- Sections
}

K. Robertson, R. Gauvin, and J. Finch

McGill University, Montreal, Quebec, Canada

Department of Mining, Metals, and Materials Engineering

The Variable Pressure Scanning Electron Microscope is one of a class of microscopes under the grouping: Gaseous Chamber SEM's. A pressurized chamber neutralizes charge accumulation on the surface of non-conducting specimens. Conductive coatings are no longer needed, thereby eliminating possible artifacts and absorption effects. Incident electron interactions with gas molecules however, degrade the beam by creating scattering events about the optical axis. Generally referred to as the skirt effect, it can hinder the ability to perform accurate x-ray microanalysis.

A correction procedure proposed by Gauvin [1], employs a linear relationship between the measured $x$-ray intensity as a function of the percent un-scattered beam $\left(f_{p}\right)$.

$$
I=\left(I_{p}-I_{m}\right) f_{p}+I_{m}
$$

Where, $\mathrm{I}$ is the intensity measured at a given pressure, $\mathrm{I}_{\mathrm{m}}$ is the Intensity at $100 \%$ scattering, $I_{p}$ is the corrected $x$-ray intensity and $f_{p}$ is the fraction of the electron beam that is not scattered. Plotting the intensity $I$ as a function of $f_{p}$ provides a linear relationship, and $I_{p}$ is obtained by extrapolation to $f_{p}=1$. This provides the real $x$-ray intensity that would be measured at high vacuum.

Experimental measurement of $\mathrm{f}_{\mathrm{p}}$ is straightforward with a dedicated sample [2], by taking the ratio of Intensity at 0 Pascals (high-vacuum) with the intensity taken at any pressure. This study uses a $\mathrm{Cu}_{2} \mathrm{~S}$ particle with a diameter smaller than 10 microns embedded in a conductive bakelite mount (Fig. 1).

Theoretical determination of $\mathrm{f}_{\mathrm{p}}$ has been prone to errors however, resulting in non-linear relationships. It has been determined that this is due to the use of the Rutherford CrossSection in the calculations. Rutherford Cross-Sections were chosen because they have been shown to be accurate for light elements at low accelerating voltages [3]. Experimental determination of the cross-sections was performed by He and Joy [4], using the technique proposed by Gauvin and Joy [5]. Comparison of Rutherford CrossSections with our experimentally measured cross-sections revealed a systematic deviation (Fig. 2). Speculation exists as to the reason for this deviation and it is likely due to an inelastic component at low accelerating voltages that have not been accounted for.

$$
f_{p}=e \frac{-0.035 * \breve{Z} * \text { Coefficient } * \mathrm{P} * \mathrm{D}}{\mathrm{TE}_{0}}
$$


Equation 2 is the revised theoretical calculation of $f_{p}$ incorporating the Cross-Section Coefficient calculated from Figure 2; which is 2.3 in our case. Comparison of the correction procedures clearly shows that the use of this correction factor for the Theoretical Cross-Section results in more accurate data (Fig. 3), providing more accurate extrapolations for the determination of the true intensity.

\section{REFERENCES}

[1] Gauvin, R., (1999). Scanning, 21, 388-393.

[2] Gauvin, R., Griffin, B., Nockolds, C., Phillips, M., Joy, D.C. (2002). Scanning, 24, 171-174.

[3] Hovington, P., Drouin, D., Gauvin, R. (1997). Scanning, 19, 1-35.

[4] He, J., and Joy, D.C. (2003). Scanning, 25(6), 285-290.

[5] Gauvin, R., and Joy, D.C. (2000). Microscopy and Microanalysis 6 (Suppl. 2), 788-789.

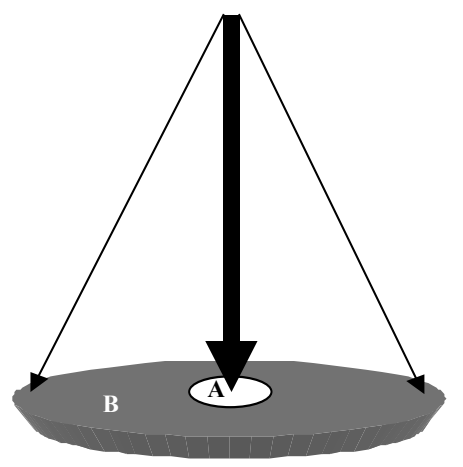

Figure 1: Geometry of experiment to measure $f_{p}$. B represents the epoxy (carbon) and $\mathrm{A}$ represents the target $\left(\mathrm{Cu}_{2} \mathrm{~S}\right)$ with diameter less than $10 \mu \mathrm{m}$. A and B must be of differing composition in order to find the percent unscattered electrons.

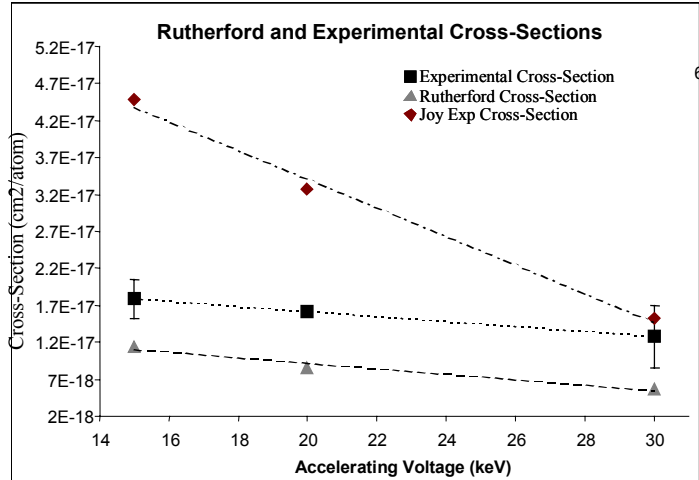

Figure 2: Comparison between Rutherford and Experimental cross-sections. There is a constant deviation between Rutherford and the Experimental Data suggesting an unknown contribution. Data from He and Joy (2003) does not match, indicating a dependency on individual microscopes.

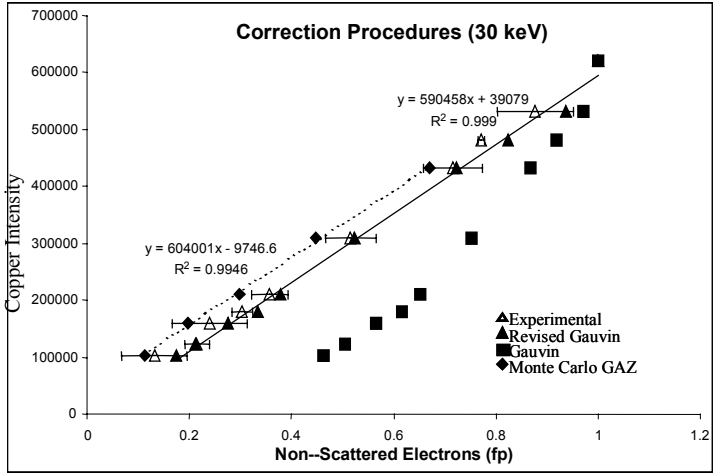

Figure 3: Plot of copper intensity versus $f_{p}$ for experimental and various theoretical models. The Revised Gauvin using a corrected Theoretical CrossSection provides the most accurate results. 